\title{
Estrabismo: detecção em uma amostra populacional e fatores demográficos associados
}

\author{
Strabismus: detection in a population-based sample and associated demographic factors
}

Augusto Tomimatsu Shimauti ${ }^{1}$, Leonardo de Toledo Pesci ${ }^{1}$, Roberta Lilian Fernandez de Sousa ${ }^{1}$, Carlos Roberto Padovani ${ }^{2}$, Silvana Artioli Schellini ${ }^{3}$

\section{RESUMO}

Objetivos: Identificar a frequência de ocorrência de desvios oculares e as características dos portadores em uma amostra populacional.

Métodos: Estudo transversal, observacional e probabilístico, entre os anos de $2004 \mathrm{e}$ 2005, envolvendo 11 cidades da região centro-oeste do estado de São Paulo. Foram examinados 10.994 indivíduos, sendo utilizada para este estudo uma subamostra desta população, identificada pelo diagnóstico de estrabismo. A população foi abordada por uma equipe treinada e padronizada para os procedimentos da pesquisa. Os dados foram analisados estatisticamente por meio de análise descritiva, frequência de ocorrência, análise de contingência e testes de associação $(p<0,05)$.

Resultados: A frequência de ocorrência de estrabismo na população estudada foi de 1,4\% (148 portadores de estrabismo), sem diferença entre sexos. Portadores de esodesvios (ET) eram 46,3\%, 38,2\% casos de exodesvio (XT) e 15,4\% de desvios verticais associados a horizontais ou síndromes. A análise de contingência mostrou que 3 indivíduos (2,3\%) estrábicos apresentavam cegueira e 7 (5,43\%) apresentavam baixa visão em um dos olhos. Tanto a ET, quanto a XT estiveram presentes em indivíduos com graus variáveis de miopia (até $-5,75$ para XT e -2,50 para ET) e de hipermetropia (até $+9,00$ para XT e $+8,00$ para ET). A associação entre estrabismo e o equivalente esférico obtido na refração estática não mostrou diferença significativa $(p>0,05)$.

Conclusão: A frequência de ocorrência de estrabismo em uma amostra populacional foi de 1,4\%, sem diferença entre sexos ou tipo de desvio ocular. A presença de cegueira e de baixa visão associadas aos desvios oculares reforçam a necessidade de tratamento precoce.

Descritores: Estrabismo/epidemiologia; Transtornos da motilidade ocular/epidemiologia

\begin{abstract}
Purposes: To identify the frequency of occurrence of ocular deviation and the carriers characteristics in a population sample.

Methods: Observational cross-sectional study, evolving a probabilistic sampling, between the years 2004 and 2005, including 11 cities of the São Paulo state. 10,994 individuals were examined. A sub-sample of this population identified as strabismus clinical diagnosis was the subject of the present study. The population was addressed by a trained team and standardized to the research procedures. The results were submitted to statistical tests through descriptive analysis, frequency of occurrence evaluation, contingency analysis and association tests $(P<0.05)$.

Results: Strabismus frequency of occurrence in the population studied was on average 1.4\% (148 strabismus carriers), with no difference between gender. Depending on the type of strabismus detected, $46.3 \%$ presented ET, 38.2\% cases of XT and $15.4 \%$ of deviations were associated with vertical or horizontal syndromes. Contingency analysis showed three ocular deviation carriers (2.3\%) with blindness (both eyes affected) and seven (5.4\%) had low vision in only one eye. According to the association of the ocular deviation and refractive error, ET and also XT can be present in individuals with varying degrees of myopia (up to -5.75 for XT and -2.50 for ET) or hyperopia (up to +9.00 for XT and +8.00 for ET). The association between strabismus and static spherical equivalent showed no significant difference between the types of eye deviation $(P>0.05)$.
\end{abstract}

Conclusion: The frequency of occurrence of strabismus in the population studied was $1,4 \%$, with no difference between gender or ocular deviation type. The presence of blindness and low vision associated to ocular deviation reinforce the need for early treatment.

Keywords: Strabismus/epidemiology; Ocular motility disorders/epidemiology

\section{INTRODUÇÃO}

O estrabismo consiste em qualquer desvio do alinhamento binocular, ou seja, ocorre quando as fóveas não estão simétricas em relação ao objeto que é focalizado pelo olhar. A classificação é de acordo com a direção do desvio em relação ao objeto de fixação, categorizando os esodesvios (ET) quando os eixos visuais estão convergentes em relação ao objeto ou ponto de fixação; exodesvio (XT) quando divergem em relação ao objeto ou ponto de fixação; hiperdesvio (HT) quando os eixos estão desviados no sentido vertical e, se mais baixo, hipotropia e hipertropia, quando mais alto em relação ao objeto de fixação(1). Porém, Bicas, na conferência do Conselho Brasileiro de Oftal- mologia de 2009, concluiu que não há uma definição estabelecida, uniforme e consistente sobre o termo "estrabismo", pois a diferença entre a normalidade e estrabismo depende, precisamente, da capacidade de colaboração do examinado para a determinação "objetiva" dos direcionamentos do olhar, por múltiplos fatores ${ }^{(2)}$.

O estrabismo pode acontecer em qualquer idade. Quando ocorre em adultos pode ser secundário a doenças neurológicas, doenças vasculares (como no diabetes melito e na hipertensão arterial), doenças da tireoide, tumores cerebrais e traumas cranianos. Pode ocorrer após perda do estímulo para fusão, devido à redução da acuidade visual (cataratas, ametropias ou outras lesões) ${ }^{(3)}$.

\footnotetext{
Submetido para publicação: 3 de maio de 2011

Aceito para publicação: 12 de janeiro de 2012

Trabalho realizado na Faculdade de Medicina de Botucatu, Universidade Estadual Paulista "Júlio de Mesquita Filho" - UNESP - Botucatu (SP), Brasil.

Médico, Departamento de Oftalmologia, Otorrinolaringologia e Cirurgia de Cabeça e Pescoço, Faculdade de Medicina, Universidade Estadual Paulista "Júlio de Mesquita Filho" - UNESP - Botucatu (SP), Brasil.

Professor, Departamento de Bioestatística, Instituto de Biociências Universidade Estadual Paulista Professor, Departamento de Bioestatística, Instituto de Biociências U
Paulista "Júlio de Mesquita Filho" - UNESP - Botucatu (SP), Brasil.

${ }^{3}$ Professor, Departamento de Oftalmologia, Otorrinolaringologia e Cirurgia de Cabeça e Pescoço, Faculdade de Medicina, Universidade Estadual Paulista Paulista "Júlio de Mesquita Filho" - UNESP .
} Botucatu (SP), Brasil.

Financiamento: Este trabalho teve financiamento da FAPESP.

Divulgação de potenciais conflitos de interesse: A.T.Shimauti, Não; L.T.Pesci, Não; R.L.F.Sousa, Não; C.R.Padovani, Não; S.A.Schellini, Não.

Endereço de correspondência: Silvana Artioli Schellini. Departamento de OFT/ORL/CCP - Faculdade de Medicina de Botucatu - UNESP - Botucatu (SP) 18618-000 - Brazil - E-mail: sartioli@fmb.unesp.br 
Se o desalinhamento do olhar ocorrer nos primeiros anos de vida, pode resultar em prejuízo para a visão, com adaptação sensorial do tipo correspondência retiniana anômala, ambliopia, ou supressão do olho que se encontra desviado. Porém, se ocorrer após o desenvolvimento da visão binocular, aparecerá diplopia e confusão de imagens ${ }^{(4)}$

Além do prejuízo funcional, com possibilidade do não desenvolvimento adequado da visão, o estrabismo muitas vezes ocasiona problemas psicológicos para o portador.

Há necessidade de se reconhecer precocemente o desvio e tratá-lo, a fim de preservar e garantir desenvolvimento adequado da visão, recuperar a visão binocular, estabelecer o paralelismo ocular e impedir a instalação de alterações psíquicas. Os esodesvios, em geral, ocorrem em crianças pequenas e os exodesvios, em adultos, associados à perda da visão por algum motivo. A depender da causa, o tratamento pode ser clínico, óptico, cirúrgico ou misto(5).

Apesar de facilmente identificáveis, a distribuição dos estrabismos na população geral não é conhecida. Também, não existem estudos que apontem qual a frequência de ocorrência ou a prevalência dos estrabismos na população brasileira.

O objetivo desde estudo consiste na identificação da frequência de ocorrência dos desvios oculares em amostra populacional da região centro-oeste paulista, assim como descrever o perfil demográfico dos portadores e a associação dos tipos de estrabismo com os erros refrativos e acuidade visual.

\section{MÉTODOS}

Foi realizado um estudo transversal, de caráter observacional e de amostragem probabilística, entre abril de 2004 e julho de 2005, envolvendo 11 cidades da região centro-oeste do estado de São Paulo, para as quais o centro de referência em saúde é a cidade de Botucatu.

Os participantes da pesquisa foram determinados por sorteio, que levou em conta o local de moradia e usando os blocos censitários do IBGE do ano 2000, sendo considerados elegíveis todos os habitantes da 5a casa do lado direito da rua e, a partir dela, assim sucessivamente. Os habitantes das moradias sorteadas recebiam uma carta-convite, explicando sobre a pesquisa que seria realizada e fornecendo a data em que seria realizado o exame. A população foi abordada usando uma Unidade Móvel Oftalmológica, equipada com dois consultórios oftalmológicos completos e com equipe que foi treinada e padronizada para a realização dos procedimentos da pesquisa. Crianças abaixo de quatro anos não tiveram o exame de acuidade visual.

O protocolo do estudo foi aprovado pelo Comitê de Ética em Pesquisa da Faculdade de Medicina de Botucatu - UNESP (Of.127/2004), e consistia de dados demográficos e semióticos com ênfase em doenças oculares (anamnese, antecedentes oculares e sistêmicos, antecedentes familiares) do participante da pesquisa.

Desta forma, foram examinados 10.994 indivíduos. Para o presente estudo, foi utilizada uma subamostra desta população, identificada pelo diagnóstico clínico de heterotropia.

O exame oftalmológico completo foi realizado pela equipe que era constituída por professores, residentes, pós-graduandos, graduandos e auxiliares de pesquisa da Faculdade de Medicina de Botucatu.

A avaliação realizada e os equipamentos empregados para a realização dos exames foram as seguintes: avaliação da acuidade visual de cada olho separadamente, com e sem o uso de correção óptica, utilizando tabelas de Snellen para cinco metros e para indivíduos iletrados; tonometria de ar (CT-60 Computerized tonometer, Topcon, Japão), biomicroscopia (lâmpada de fenda DFVasconcelos, Brasil), fundoscopia direta (em lâmpada de fenda usando lente de Volk de 78 dioptrias ou oftalmoscópio direto Welch Allen, USA), fundoscopia indireta (com oftalmoscópio binocular indireto Welch Allen, USA, e lente de 20 dioptrias), realizada em casos especiais; exame refracional sob cicloplegia (uma gota de colírio de ciclopentolato 1\%, exami- nando-se 30 minutos após a instilação) quando se tratava de pessoas com menos do que 40 anos de idade. Para o exame refracional o indivíduo foi examinado em refrator automático (ShinnNippon, Japão) para avaliação da refração objetiva, seguindo-se da refração subjetiva, realizada em refrator manual (Nidek, Japão).

Especificamente para diagnóstico dos estrabismos foram realizados os testes de Hirschberg, teste de cobertura simples e teste de cobertura alternado, categorizando o indivíduo em ortotrópico (quando os desvios estavam ausentes) ou portadores de heterotropias: ET, XT ou desvios associados (estrabismos verticais e horizontais associados ou síndromes), conforme apontado pelos testes de avaliação para detecção de desvios oculares.

Os resultados foram transferidos para a planilha eletrônica Microsoft Excel $2002^{\circledR}$ e submetidos a testes estatísticos por meio de análise descritiva, avaliação de frequência de ocorrência e análise de contingência (teste de valor P e teste de Goodman). Para análise de associação de erros refrativos com os tipos de estrabismo, utilizou-se - grau esférico, o cilíndrico e o equivalente esférico das refrações realizadas sob cicloplegia. A significância foi apontada para $p<0,05$.

\section{RESULTADOS}

A frequência de ocorrência de estrabismo na população estudada, considerando a média obtida em todas as 11 cidades avaliadas, foi de 1,4\%. A distribuição dos porcentuais de estrábicos em relação ao município avaliado encontra-se na tabela 1, onde se nota que a variação de heterotrópicos detectados nos diferentes municípios foi de $0,9 \%$ até $1,6 \%$.

As análises foram realizadas utilizando os dados dos portadores de heterotropia que eram em número de 148 indivíduos, distribuídos em 71 (48\%) do sexo masculino e 77 (52\%) do feminino. A adesão para participação no estudo foi maior entre as mulheres (52\%), o que foi considerado para o cálculo de diferença de ocorrência de heterotropia entre os sexos, observando-se que ambos os sexos foram afetados na mesma proporção.

Apesar do número de heterotrópicos detectados ter sido de 148, para a análise de algumas variáveis dados perdidos levaram a diferentes totalizações, fato considerado quando da aplicação dos testes estatísticos.

Segundo o tipo de heterotropia detectada, 46,3\% eram portadores de ET, 38,2\% casos de XT e 15,4\% de desvios verticais associados a horizontais ou síndromes, com possibilidade semelhante para o encontro de ET ou XT $(p=0,5)$ na população estudada (Tabela 2).

$\begin{aligned} & \text { Tabela 1. Distribuição do total de examinados e dos portadores de } \\
& \text { estrabismo nos municípios da região centro-oeste do estado de São } \\
& \text { Paulo e que fizeram parte do estudo }\end{aligned}$
\begin{tabular}{lcc}
\hline Município & Total de examinados & Porcentual de estrábicos* \\
\hline Arandu & 746 & 1,2 \\
Areiópolis & 756 & 0,9 \\
Bofete & 690 & 1,6 \\
Conchas & 1.011 & 1,3 \\
Itaí & 1.018 & 1,5 \\
Manduri & 1.020 & 1,0 \\
Pereiras & 895 & 0,9 \\
Pratania & 695 & 1,4 \\
Taguaí & 813 & 1,6 \\
Botucatu & 2.552 & 1,5 \\
Piraju & 798 & 1,6 \\
\hline
\end{tabular}

*=os porcentuais foram calculados em relação ao número total de indivíduos examinados em cada município 
Utilizando o teste de Goodman para a análise do tipo de heterotropia em relação à acuidade visual no pior olho, a comparação entre os tipos de heterotropia mostra que a maioria $(50,9 \%)$ dos portadores de ET apresentava acuidade visual corrigida melhor que 0,7 e que os portadores de desvios associados foram os que mais apresentaram acuidade visual entre 0,3 e 0,05 (Tabela 3). Dentro dos grupos de tipos de heterotropia, foi possível observar que a chance de se encontrar indivíduos com acuidade visual acima de 0,7 no pior olho é maior em portadores de ET (Tabela 3).

A análise de contingência mostra que $48,1 \%$ dos portadores de heterotropia apresentavam acuidade visual corrigida melhor que 0,7 em ambos os olhos. A acuidade visual corrigida foi menor que 0,3 e maior que 0,05 (definição adotada pela OMS para deficiência visual) em 3,9\% dos portadores de desvios oculares, dos quais $7(5,4 \%)$ apresentavam baixa visão em apenas um dos olhos. Três indivíduos estrábicos (2,3\%) apresentavam acuidade visual corrigida compatível com cegueira bilateral (seguindo-se a definição da OMS) (Tabela 4).

As medidas descritivas dos equivalentes esféricos obtidos das refrações estáticas, construídos a partir dos erros refrativos e a associação com os tipos de heterotropia, estão apresentadas na tabela 5, onde é possível observar que todos os tipos de desvios oculares podem estar presentes em indivíduos com graus variáveis de miopia (até $-5,75$ para XT e -2,50 para ET) e de hipermetropia (até +9,00 para XT e $+8,00$ para ET). Portanto, para a população estudada, a avaliação de associação entre heterotropia e o equivalente esférico obtido no exame refracional não mostrou diferença significativa entre os diferentes tipos de desvio ocular $(p>0,05)$.

\section{DISCUSSÃO}

O presente estudo oferece a oportunidade de conhecer características dos desvios oculares em amostra populacional brasileira, sendo esta proveniente da região centro-oeste do estado de São Paulo. Não há outros estudos semelhantes publicados sobre o assunto. As pesquisas existentes são relativas a segmentos de faixas etárias específicas, sendo a maioria infanti ${ }^{(4,6,7)}$ e um em idosos ${ }^{(8)}$, realizados em amostras de conveniência. Embora alguns incluam todas as faixas etárias, são estudos retrospectivos e realizados utilizando dados de indivíduos atendidos em consultório ${ }^{(9,10)}$, não se tratando de amostras probabilísticas.

A prevalência de estrabismo na população estudada foi, em média, de 1,4\%, com distribuição semelhante em todos os municípios avaliados. Este número concorda com os números mundiais sobre a frequência de heterotropia em crianças, que varia de $0,12 \%$ a 2,7\% ${ }^{(7,11)}$.
Não houve diferença significativa de existência de estrabismo entre os sexos como encontrado em outros trabalhos ${ }^{(4,11,12)}$, apesar do estudo feito em São Paulo ter apontado para predomínio de XT no sexo feminino ${ }^{(5)}$.

As prevalências das ETs são maiores nas populações européias ou seus descendentes e as XTs, mais nos asiáticos, como comprovado por vários artigos ${ }^{(10,13,14)}$. No presente estudo, 46,3\% eram portadores de ETs e 38,2\%, de XTs, sendo a maioria da população que participou da presente pesquisa autorreferida como portadora de cor da pele branca. Contudo, é necessário frisar que a população brasileira é bastante miscigenada, não sendo possível afirmar dados sobre determinada raça no Brasil. Outro estudo também mostra prevalência semelhante, sendo $44,5 \%$ portadora de $\mathrm{ET}^{(5)}$, número muito próximo do aqui detectado.

Embora as ETs sejam mais relacionadas com distúrbios visuais, como visto em estudo retrospectivo realizado em amostra de conveniência de nosso serviço que apontou haver maior relação de ET com a ambliopia ${ }^{(15)}$, no presente estudo que analisa os estrabismos na população geral e não em amostra que se apresenta em serviço de referência, a maioria dos portadores de heterotropia teve acuidade visual no pior olho maior que 0,7 , sendo que foram os desvios associados os que mais se apresentaram com acuidade visual reduzida.

Classicamente, determinados tipos de heterotropia são mais associados a algum erro refrativo específico, como por exemplo, as ETs acomodativas associadas com altos graus de hipermetropia. Entretanto, no presente estudo onde não se categorizou os diferentes tipos de ETs ou XTs, verificou-se que não houve diferença significativa entre os desvios oculares relacionados com o equivalente esférico do erro refrativo (miopia ou hipermetropia), que estiveram presentes tanto nas ETs, como nas XTs, apresentando-se com ampla faixa de variação.

A análise de contingência mostrou que quase 50\% dos estrábicos apresenta boa acuidade visual em ambos os olhos. Os heterotrópicos quando apresentam comprometimento da visão, encontram-se na faixa de deficiência visual (considerando as definições da OMS para deficiência visual). Em geral, a baixa visão ocorre em um dos olhos, concordando com outro estudo que mostrou que a anisometropia e o estrabismo são responsáveis por deterioração visual uniocular, mas não deficiência visual(16), embora tenha sido identificada deficiência visual bilateral em 3,9\% dos estrábicos avaliados.

A cegueira em ambos os olhos foi demonstrada pela tabela de contingência como presente em 2,3\% dos casos (considerando a

Tabela 2. Distribuição dos portadores de estrabismo de acordo com o sexo e tipo de desvio

\begin{tabular}{|c|c|c|c|c|}
\hline \multirow[b]{2}{*}{ Sexo } & \multicolumn{3}{|c|}{ Diagnóstico } & \multirow[b]{2}{*}{$\begin{array}{l}\text { Total } \\
\text { n (\%) } \\
\end{array}$} \\
\hline & $\begin{array}{c}\text { Desvio associado } \\
\text { n (\%) }\end{array}$ & $\begin{array}{c}\mathrm{XT} \\
\mathrm{n}(\%)\end{array}$ & $\begin{array}{c}\text { ET } \\
\text { n (\%) }\end{array}$ & \\
\hline Masculino & $14(22,6)$ & $19(30,6)$ & $29(46,8)$ & $62(100)$ \\
\hline Feminino & $5(8,2)$ & $28(45,9)$ & $28(45,9)$ & $61(100)$ \\
\hline Total & $19(15,4)$ & $47(38,2)$ & $57(46,3)$ & $123(100)$ \\
\hline
\end{tabular}

Tabela 3. Distribuição dos tipos de estrabismo em relação à acuidade visual corrigida no pior olho

\begin{tabular}{lccccc}
\hline & \multicolumn{4}{c}{ Acuidade visual } & \multirow{2}{*}{ Total } \\
\cline { 2 - 5 } Diagnóstico & $\mathbf{2 0 , 7}$ & $\mathbf{0 , 7} \mathbf{a} \mathbf{0 , 3}$ & $\mathbf{0 , 3} \mathbf{a} \mathbf{0 , 0 5}$ & $<\mathbf{0 , 0 5}$ & \\
\hline Desvio associado & $3(15,8) \mathrm{aA}$ * & $3(15,8) \mathrm{aA}$ & $8(42,1) \mathrm{bA}$ & $5(26,3) \mathrm{aA}$ & 19 \\
Exotropia & $14(31,8) \mathrm{abA}$ & $13(29,5) \mathrm{aA}$ & $5(11,4) \mathrm{aA}$ & $12(27,3) \mathrm{aA}$ & 44 \\
Esotropia & $27(50,9) \mathrm{bB}$ & $8(5,1) \mathrm{aA}$ & $8(15,1) \mathrm{abA}$ & $10(18,9) \mathrm{aA}$ & 53 \\
\hline
\end{tabular}

* = teste de Goodman: letras minúsculas comparam dados na vertical e as maiúsculas na horizontal, de modo que duas letras diferentes indicam significância estatística 
Tabela 4. Análise de contingência para verificação de associação da acuidade visual no olho direito e no olho esquerdo em portadores de estrabismo - UNESP, 2011

\begin{tabular}{|c|c|c|c|c|c|}
\hline \multirow[b]{2}{*}{ Olho direito } & \multicolumn{4}{|c|}{ Olho esquerdo } & \multirow[b]{2}{*}{ Total } \\
\hline & $\geq 0,7$ & 0,3 a 0,7 & 0,05 a 0,3 & $<0,05$ & \\
\hline$\geq 0,7$ & $62(48,1)$ & $7(5,4)$ & $8(6,2)$ & $7(5,4)$ & $84(65,1)$ \\
\hline 0,3 a 0,7 & $9(7,0)$ & $6(4,6)$ & $0(0,0)$ & $4(3,1)$ & $19(14,7)$ \\
\hline 0,05 a 0,3 & $8(6,2)$ & $3(2,3)$ & $5(3,9)$ & $0(0,0)$ & $16(12,4)$ \\
\hline$<0,05$ & $4(3,1)$ & $1(0,8)$ & $2(1,5)$ & $3(2,3)$ & $10(7,7)$ \\
\hline Total & $83(64,4)$ & $17(13,1)$ & $15(11,6)$ & $14(10,8)$ & $129(100)$ \\
\hline
\end{tabular}

Tabela 5. Medidas descritivas referentes aos equivalentes esféricos construídos a partir dos erros refrativos estáticos observados, em relação aos tipos de estrabismo

\begin{tabular}{|c|c|c|c|c|}
\hline \multirow{2}{*}{$\begin{array}{l}\text { Medida descritiva } \\
\text { equivalente esférico }\end{array}$} & \multicolumn{3}{|c|}{ Diagnóstico } & \multirow[b]{2}{*}{ Valor de $p$} \\
\hline & Desvio associado & $\mathbf{X T}$ & ET & \\
\hline Valor mínimo & $-4,25$ & $-5,75$ & $-2,50$ & \\
\hline 1ㅇquartil & 0,00 & 0,00 & 0,00 & \\
\hline Mediana & 1,13 & 0,13 & 0,00 & \\
\hline 3ㅇquartil & 2,50 & 1,00 & 1,00 & $p>0,05$ \\
\hline Valor máximo & 7,00 & 9,00 & 8,00 & \\
\hline Média & 1,29 & 0,76 & 1,32 & \\
\hline Desvio padrão & 2,93 & 2,78 & 2,42 & \\
\hline
\end{tabular}

definição da OMS que considera a acuidade visual menor que 0,05 para a definição de cegueira).

Outro estudo mostrou a taxa de cegueira (com a acuidade visual avaliada utilizando a melhor correção óptica) para a população geral da mesma região abrangida por este estudo como de 0,4\%(17). Assim, o encontro de número muito superior de cegos entre os portadores de estrabismo poderia significar que a causa dos desvios oculares nos portadores de acuidade visual reduzida em ambos os olhos poderia ser a perda dos mecanismos fusionais, dependentes da boa visão em ambos os olhos.

No ano de 2007, um estudo feito em um campo de refugiados no Sudão com uma realidade extremamente carente de cuidados médicos, mostrou $2,7 \%$ de cegueira e 1,6\% de deficiência visual grave em crianças ${ }^{(18)}$, números próximos aos detectados como portadores de cegueira em indivíduos com desvio ocular na nossa população de estrábicos.

É importante frisar que o estrabismo não tem relação com a condição social, como já observado por outros ${ }^{(12)}$, mas, sim, às consequências da sua não abordagem clínica ou cirúrgica.

Apesar dos bons indicadores sociais presentes nos municípios brasileiros que fizeram parte desta pesquisa, uma constatação muito frequente foi que os portadores de estrabismo estavam sem tratamento, muitos portadores de ambliopia já irreversível ou de desvios bastante inestéticos, por falta de oportunidade de acesso aos serviços de atendimento gratuito (SUS), o que deve servir de ponto de reflexão para os gestores. Desta forma, os nossos dados confirmam que a acessibilidade ao tratamento desta afecção que é de simples e fácil diagnóstico, ainda é precária no nosso meio.

\section{CONCLUSÃO}

A frequência de ocorrência de estrabismo na população centrooeste do Estado de São Paulo foi de 1,4\%, acometendo igualmente ambos os sexos, sendo os desvios convergentes e os desvios divergentes encontrados em proporções semelhantes. Os autores cha- mam a atenção para os índices de deficiência visual (3,9\%) e cegueira $(2,3 \%)$ observados em portadores de uma afecção que é de simples detecção e que necessita de tratamento precoce para ter solução favorável, fazendo reflexões sobre a acessibilidade ao tratamento como fonte para os resultados desfavoráveis.

\section{AGRADECIMENTO}

Agradecemos aos Drs. Keiti Fernando Shirasu e Fabrício Rendeiro Sales pelo auxílio no levantamento dos dados utilizados na presente pesquisa.

\section{REFERÊNCIAS}

1. Spalton DJ, Hitchings RA, Hunter PA. Atlas de oftalmologia clínica. 3a ed. Rio de Janeiro: Elsevier, 2006. p.603-35.

2. Bicas HEA. Estrabismos: da teoria à prática, dos conceitos às suas operacionalizações. Arq Bras Oftalmol. 2009;72(5):585-615.

3. Prieto-Díaz J, Souza-Dias C. Estrabismo. 4a ed. São Paulo: Livraria Santos Editora; 2002. p.149-50.

4. Garcia CAA, Sousa AB, Mendonça MBM, Andrade LL, Oréfice F. Prevalence of strabismus among students in Natal/RN - Brazil. Arq Bras Oftalmol. 2004;67(5):791-4.

5. Kac MJ, Freitas Júnior MB, Kac SI, Andrade EP. Frequência dos tipos de desvios oculares no ambulatório de motilidade ocular extrínseca do Hospital do Servidor Público Estadual de São Paulo. Arq Bras Oftalmol. 2007;70(6):939-42.

6. Friedman Z, Neumann E, Hyams SW, Peleg B. Ophthalmic screening of 38,000 children, age 1 to $2 \frac{1}{2}$ years, in child welfare clinics. J Pediatr Ophthalmol Strabismus. 1980; 17(4):261-7.

7. Matsuo T, Matuo C, Matsuoka H, Kio K. Detection of strabismus and amblyopia and amblyopia in 1.5- and 3-year-old children by a preschool vision-screening program in Japan. Acta Med Okayama. 2007:61(1):9-16.

8. Magramm I, Schlossman A. Strabismus in patients over the age of 60 years. J Pediatr Ophthalmol Strabismus. 1991;28(1):28-31.

9. Sá LCF. Estrabismo nos anos 90. Arq Bras Oftalmol. 1998;61(6):720-4.

10. Yu CB, Fan DS, Wong VW, Wong CY, Lam DS. Changing patterns of strabismus: a decade of experience in Hong Kong. Br J Ophthalmol. 2002;86(8):854-6. Comment in $\mathrm{Br}$ J Ophthalmol. 2002;86(8):835-6.

11. Robaei D, Kifley A, Mitchell P. Factors associated with a previous diagnosis of strabismus in a population-based sample of 12-year-old Australian children. Am J Ophthalmol. 2006;142(6):1085-8. 
12. Graham PA. Epidemiology of strabismus. Brit J Ophthalmol. 1974;58(3):224-31.

13. Chia A, Dirani M, Chan YH, Gazzard G, Eong KG, Selvaraj P, et al. Prevalence of amblyopia and strabismus in young singaporean chinese children. Invest Ophthalmol Vis Sci. 2010;51(7):3411-7.

14. Mohney BG. Common forms of childhood strabismus in an incidence cohort. Am J Ophthalmol. 2007;144(3):465-7.

15. Gonçalves F, Schellini SA, Heimbeck FJ, Furuya MT, Padovani CR. Causas de ambliopia e resultados e tratamento. Rev Bras Oftalmol. 2006;65(2):104-8.
16. Moll A, Orawiec B, Niwald A, Gralek M. Causes of visual disability in children and young adults. Klin Oczna. 2005;107(1-3):93-5

17. Schellini SA, Durkin SR, Hoyama E, Hirai F, Cordeiro R, Casson RJ, et al. Prevalence and causes of visual impairment in a Brazilian population: the Botucatu Eye Study. BMC Ophthalmol. 2009:9:8.

18. Zeidan Z, Hashim K, Muhit MA, Gilbert C. Prevalence and causes of childhood blindness in camps for displaced persons in Khartoum: results of a household survey. East Mediterr Health J. 2007;13(3):580-5.

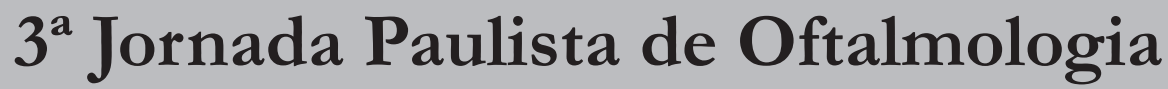

\author{
22 e 23 de junho de 2012 \\ Vitória Hotel Concept - Campinas (SP) \\ Realização: UNESP (Botucatu) \\ UNICAMP (Campinas) \\ USP (Ribeirão Preto)
}

Informações:

JDE - Organização de Eventos

Tel.: (11) 5084-9174 / 5084-5284 - Fax: (11) 5574-8261

E-mail: secretaria.jpo@jdeeventos.com.br

Site: www.jornadapaulistadeoftalmo.com.br 